\title{
Evaluación electroquímica de soldaduras en tubos capilares de acero inoxidable
}

\section{Electrochemical evaluation of stainless steel welded tubes}

Javier E. Rodríguez-Yáñez'

Ericka Saborío-Leiva²

Daniel Mora-Montoya ${ }^{3}$

Rodríguez-Yáñez, j; Saborío-Leiva, E; MoraMontoya, D. Evaluación electroquímica de soldaduras en tubos capilares de acero inoxidable. Tecnología en Marcha. Vol. 27, Nº 


\section{Palabras clave}

Acero inoxidable 316L; soldadura orbital autógena; Tafel; geotermia; decapado.

\section{Resumen}

La recuperación de capilares de acero 316L utilizados en pozos geotérmicos neutros, por medio de soldadura orbital autógena, se evalúa desde el punto de vista de su resistencia a la corrosión en las condiciones de utilización, mediante técnicas electroquímicas básicas.

En el pretratamiento se encuentra que es más conveniente realizar un decapado químico previo a la soldadura por 5 minutos. Mientras que posteriormente a la soldadura, la aplicación de tratamiento térmico de reducción de tensiones no se justifica desde el punto de vista de la resistencia a la corrosión.

También se plantea la comparación de los capilares soldados respecto a capilares nuevos y usados, teniendo los capilares soldados un comportamiento intermedio entre ambos.

\section{Key words}

3 I 6L Steel; orbital welding; Tafel; geothermal; pickling.

\section{Abstract}

The recovery of 316L steel capillaries used in neutral geothermal wells orbital welding by weld is evaluated from the point of view of its resistance to corrosion in the conditions of use, through basic electrochemical techniques.

In the pre-treatment is more convenient than carrying out a chemical pickling prior to welding for 5 minutes. While subsequent to the application of welding heat treatment of stress reduction is not justified from the standpoint of corrosion resistance.

There is also the comparison of capillaries capillary soldiers regarding new and used soldier's capillaries having an intermediate behavior between the two.

\section{Introducción}

El uso de los recursos geotérmicos para generar electricidad se ha incrementado en los últimos 20 años, siendo su principal desarrollador en Costa Rica el Centro de Servicio de Recursos Geotérmicos (CSRG) del Instituto Costarricense de Electricidad (ICE). El proceso implica la extracción de aguas y vapor sobrecalentados, mediante pozos en acuíferos profundos calentados por la actividad magmática. La estabilidad del proceso y su continuidad es una de las características principales de la generación geotérmica. Esa estabilidad depende de las políticas de explotación y del control de fenómenos secundarios como la formación de depósitos de carbonato de calcio $\left(\mathrm{CaCO}_{3}\right)$, que ocurre en la zona de ebullición de los pozos neutros como resultado de la sobresaturación de bicarbonatos $\left(\mathrm{HCO}_{3}\right)$.
Para evitar la formación de incrustaciones de carbonatos de calcio, en los pozos geotérmicos neutros se recurre a la aplicación de inhibidores en el fondo de estos. En general, estos inhibidores son compuestos de tipo poliacrilato. Para su aplicación se utilizan tubos capilares continuos de acero inoxidable AISISAE 316L, que resisten mejor las características químicas de los fluidos con $\mathrm{pH}$ neutros. La durabilidad de dichos capilares depende de muchas variables pero en general se les estima una vida útil de I,5 a 3 años. Los capilares que se pueden recuperar son los que presentan obstrucciones debido a la cristalización de los inhibidores, no aquellos que fallan por esfuerzo o por corrosión bajo tensión (stress corrosion cracking o SCC). Es por eso que se plantearon metodologías de soldado de dichos capilares y sus condiciones comparativas de solda- 
dura respecto a un capilar nuevo y un capilar usado, evaluándose las metodologías mediante ensayos electroquímicos.

El estudio del comportamiento del acero AISI-SAE 316L bajo las condiciones establecidas en este artículo es un tema muy específico. Se encontró que la bibliografía se enfoca en la corrosión en los procesos geotérmicos y otros en aceros de distintos tipos no siempre asociados a pruebas electroquímicas (Ellis, 1985; XU et. al, 2006; Whittake, 2009).

El acero AISI-SAE 3|6L, al utilizarse en procesos donde se incrementa el riesgo de sufrir corrosión, ha sido estudiado por medio de ensayos electroquímicos y gravimétricos, junto a otras aleaciones, para observar su desempeño. Entre los medios utilizados en las pruebas de corrosión se encuentran las salmueras geotérmicas, 2-Mercaptobenzimidazole y soluciones de bacteria oxidante de hierro y de bacteria reductora de sulfato (Mundhenk et. al, 20 I 3; $X U$ et. al, 2006).

Las salmueras geotérmicas varían según la naturaleza del yacimiento que les da origen, por lo tanto las comparaciones indicadas aquí deben ser valoradas en cada situación y siempre asociada a pozos geotérmicos de $\mathrm{pH}$ neutros (Ellis, 1985; Mora, 2006).

\section{Materiales y metodología}

Los capilares de acero inoxidable AISI- SAE 316L, con un diámetro exterior de 6,35 mm y I,24 mm de espesor, utilizados en los pozos neutros del Campo Geotérmico Miravalles presentan la siguiente composición química, según los proveedores del CSRG (Mora Rodriguez, 2006).

\section{Disoluciones}

Antes del soldado de los capilares se efectúa una limpieza con acetona grado reactivo (95\%) para la remoción de grasas o hidrocarburos, luego se aplica un decapado según la norma ASTM A 380-06, Sección 7.

Cuadro 1. Composición química porcentual de los capilares de acero inoxidable AISI- SAE 316L.

\begin{tabular}{|c|c|c|c|c|c|c|}
\hline Elemento & $\mathrm{C}$ & $\mathrm{Si}$ & $\mathrm{Mn}$ & $\mathrm{Cr}$ & $\mathrm{Ni}$ & $\mathrm{Mo}$ \\
\hline Porcentaje & 0,03 & 0,6 & 1,7 & 17,5 & 13 & 2,6 \\
\hline
\end{tabular}

En este estudio, el decapado se realizó con una disolución preparada a partir de $\mathrm{HNO}_{3}$ concentrado $\left(70 \% \mathrm{~m} / \mathrm{m}\right.$ y densidad $\left.\mathrm{I}, 4 \mathrm{I} \mathrm{g} / \mathrm{cm}^{3}\right)$ y HF concentrado $\left(48 \% \mathrm{~m} / \mathrm{m}\right.$ y densidad $\left.1,15 \mathrm{~g} / \mathrm{cm}^{3}\right)$, a fin de obtener una relación $24 / 5 \% \mathrm{~m} / \mathrm{v}$. Las muestras de acero inoxidable AISI-SAE3I6L fueron decapadas con dicha disolución a tiempos de 2, 5, 8, 10, 15, 20, 25, 30 minutos y comparadas con muestras del mismo acero nuevas y usadas (Mora Rodriguez, 2006; ASME, 2009; ASTM A 407, 2007).

Se utilizó una disolución reveladora de Ferroxil para obtener una referencia sobre la acumulación de óxido en la superficie del tubo capilar, siguiendo la norma ASTM A 380-06, Sección 7. La disolución se preparó con $30 \mathrm{~g}$ de $\mathrm{K}_{4}\left[\mathrm{Fe}(\mathrm{CN})_{6}\right](99 \%), 17,5 \mathrm{~cm}^{3}$ de $\mathrm{HNO}_{3}$ concentrado y agua destilada c.s.p. 1000 $\mathrm{cm}^{3}$ (Mora Rodríguez, 2006; Davies et. al, 1998).

Posteriormente al decapado y soldado, las muestras se sometieron a tratamientos térmicos de alivio de tensiones a $410^{\circ} \mathrm{C}$ y $955^{\circ} \mathrm{C}$. Los tratamientos térmicos (TT) se realizaron en un horno Lindberg Blue M $1200^{\circ} \mathrm{C}$, modelo HTF55342C, durante 12 minutos. Los resultados se compararon con las muestras sin TT (Mora Rodríguez, 2006; Davies et. al, 1998).

Para el proceso de evaluación electroquímica del decapado se utilizó como electrolito una disolución neutra de $\mathrm{NaNO}_{3}$ al $10 \% \mathrm{~m} / \mathrm{v}$, a partir de reactivo Sigma puro (99,5\%), mientras que para los capilares soldados se usaron las disoluciones a las que están expuestos habitualmente en los pozos geotérmicos neutros: solución de poliacrilato de sodio al $5 \%$ y la salmuera neutra del pozo $\mathrm{N}^{\circ} 29$.

La composición obtenida del proveedor respecto al poliacrilato solo indica que es una mezcla de ácidos policarboxilicos de sodio de la Bio-LabWater Additives Division (producto DP 3537) (Davies et. al, 1998). La salmuera del Pozo 29 presenta una composición química neutra, con valores medios de cloruros y sulfatos, pero con la mayor cantidad de bicarbonatos, con posibilidad de formación de incrustaciones. La composición de su fase líquida puede verse en el cuadro 2.

Todas las disoluciones se elaboraron con aguas de calidad tipo IV según la norma ASTM D I 193-06 (con conductividad menor a $5 \mu \mathrm{S} / \mathrm{cm}, \mathrm{pH}$ entre 5 y 8, así como concentraciones de $\mathrm{Na}$ y $\mathrm{Cl}$ menores a $50 \mathrm{mg} / \mathrm{l})$ (ASTM D | 193, 20 I I). 
Cuadro 2. Composición de la salmuera neutra del pozo PGM N²9, medidas de la fase líquida a $98^{\circ} \mathrm{C}$

\begin{tabular}{|c|c|}
\hline Pozo & PGM-29 \\
\hline Fecha & $20-$ feb- 12 \\
\hline $\mathrm{pH}$ & 7,35 \\
\hline Cond. $(\mu \mathrm{S} / \mathrm{cm})$ & 13930 \\
\hline $\mathrm{Na}$ (ppm) & 2838 \\
\hline K (ppm) & 256 \\
\hline Ca (ppm) & 79 \\
\hline Mg (ppm) & 0,21 \\
\hline Fe total.(ppm) & $<0,07$ \\
\hline $\mathrm{Cl}$ (ppm) & 4508 \\
\hline $\mathrm{SO}_{4}(\mathrm{ppm})$ & 74 \\
\hline $\mathrm{HCO}_{3}$ (ppm) & 161 \\
\hline$F(p p m)$ & 2,43 \\
\hline B (ppm) & 66 \\
\hline $\mathrm{H}_{2} \mathrm{~S}$ (ppm) & $<1$ \\
\hline $\mathrm{NH}_{3}(\mathrm{ppm})$ & 1,12 \\
\hline As (ppm) & 10,0 \\
\hline $\mathrm{SiO}_{2}$ monomérica (ppm) & 563 \\
\hline $\mathrm{SiO}_{2}$ Total (ppm) & 591 \\
\hline S.T.D (ppm) & 8700 \\
\hline
\end{tabular}

\section{Ensayos electroquímicos}

Las metodologías utilizadas para la evaluación de la corrosión son las siguientes ${ }^{4}$ (Fontana, 1986; ASTM G I02, 20।0; ASTM G 5, 2004):

I. Potencial de circuito abierto $\left(E_{o c}\right)$, durante 10 minutos.

2. Resistencia de polarización lineal $\left(R_{\mathrm{p}}\right)$, mediante la aplicación de un barrido de potencial a 10 $\mathrm{mV} / \mathrm{min}$ entre $-20 \mathrm{mV} y+20 \mathrm{mV}$ respecto a $E_{o c}$.

3. Curvas de Tafel catódica y anódica, con un barrido de potencial de $50 \mathrm{mV} / \mathrm{min}$, entre -250 $m V y+250 m V$, respecto a $E_{o c}$

$4 \quad$ Hoja técnica y MSDS del inhibidor DP 3537 Mixture of PolycarboxylicAcids (Poliacrilato de sodio) BioLabß.
4. Curvas de Tafel anódicas cíclicas, con un barrido de potencial de $50 \mathrm{mV} / \mathrm{min}$, entre $0 \mathrm{mV}$ a $+1000 m V$ respecto $E_{o c^{\prime}}$ para los capilares soldados con $T$.

Las evaluaciones se realizaron a temperatura ambiente $\left(20^{\circ} \mathrm{C}\right)$, mientras que el electrodo de referencia utilizado es el de Calomel (SCE) y el contraelectrodo es de platino (Pt).Todos los potenciales son referidos al electrodo de SCE.

Para realizar las mediciones electroquímicas se utilizó un potenciostato-galvanostato marca Gamry, modelo Reference 600. Las velocidades de corrosión $\left(V_{\text {corr }}\right)$ se calculan a partir de los valores de Rp, las áreas expuestas y las pendientes de Tafel, según la ecuación general de Stern- Geary (Fontana, 1986; Bard et al, 200 I; Schweitzer, 1986).

\section{Metalografías}

Se realizó la evaluación metalográfica de los capilares de acero inoxidable 316L, inspeccionando la soldadura, zona afectada térmicamente, y el material base.

Las probetas fueron pulidas y atacadas electrolíticamente, usando como electrolito de pulido una solución de etanol al 95\%, agua desionizada y ácido perclórico al 60\%, con densidad I,5 g/ $\mathrm{cm}^{3}$ (relación $40 / 7 / 3$ v/v) durante $40 \mathrm{~s}$ a $60 \mathrm{~V}$. Como reactivo de ataque se utilizó una solución de ácido nítrico concentrado (60\% y densidad: I, $37 \mathrm{~g} / \mathrm{cm}^{3}$ ) durante 120 s a I V (ASM International Metal Handbook, 1990; González, 1989).

El análisis se efectuó utilizando un microscopio metalográfico marca Olympus, modelo GX4I, y el software analizador de imágenes Paxit IA44, marca Leco.

\section{Resultados y discusión}

\section{Proceso de decapado}

Los valores generales obtenidos pueden apreciarse en el cuadro 3. En la mezcla decapante, el ácido nítrico $\left(\mathrm{HNO}_{3}\right)$ actúa como un oxidante de la superficie facilitando la formación de películas protectoras, mientras que el ácido fluorhídrico (HF) actúa como un ácido reductor eliminando los óxidos superficiales existentes y atacando el material base (Davies, 1998; Laugfgang, 2000). En ellas se observó que a partir de los 2 minutos de decapado, no se obser- 
Cuadro 3. Valores obtenidos en los ensayos electroquímicos con solución de $\mathrm{NaNO}_{3}$ al $10 \%$ sobre capilares de acero 316L nuevos, usados y decapados a distintos tiempos.

\begin{tabular}{|c|c|c|c|}
\hline $\begin{array}{l}\text { Tiempo de } \\
\text { decapado }\end{array}$ & $\begin{array}{c}E_{o c} \\
(m \vee v s S C E)\end{array}$ & $\begin{array}{c}V_{\text {corr }} \\
(\mu \mathrm{m} / \mathrm{año})\end{array}$ & $\begin{array}{c}V_{\text {corr }} \\
\left(g / m^{2} \text { año }\right)\end{array}$ \\
\hline Nuevo & $-386,1$ & 0,135 & I,0829 \\
\hline Usado & 232,7 & 0,067 & 0,5368 \\
\hline $2 \mathrm{~min}$ & 27,94 & 0,100 & 0,8022 \\
\hline $5 \mathrm{~min}$ & $-0,06$ & 0,088 & 0,7020 \\
\hline $8 \mathrm{~min}$ & $-4,9$ & 0,053 & 0,4257 \\
\hline $10 \mathrm{~min}$ & $-10,28$ & 0,065 & 0,5203 \\
\hline $15 \mathrm{~min}$ & $-61,48$ & 0,120 & 0,9626 \\
\hline $20 \mathrm{~min}$ & $-153,5$ & 0,112 & 0,8962 \\
\hline $25 \mathrm{~min}$ & $-155,4$ & 0,140 & I, 1202 \\
\hline $30 \mathrm{~min}$ & $-181,1$ & 0,171 & 1,3663 \\
\hline
\end{tabular}

van más residuales de óxidos de hierro en la superficie que reaccionen con el reactivo de Ferroxil.

El proceso de decapado modifica los valores de Eoc, disminuyéndolos con el tiempo de decapado sobre el capilar usado, lo cual puede apreciarse en la figura I. Los valores obtenidos son en todos los casos superiores, o más positivos, que los de un capilar nuevo, pero menores que el capilar usado, lo que indicaría un aumento de estabilidad de la superficie frente a la corrosión por el proceso de decapado en condiciones estáticas.

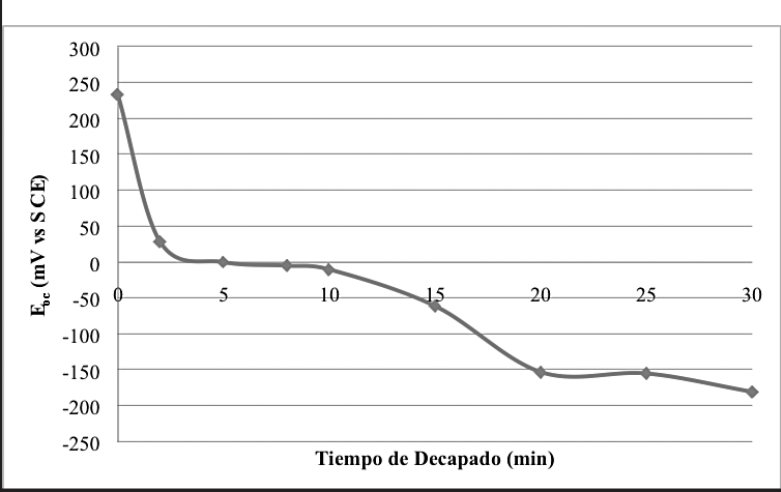

Figura I. Variación del potencialde circuito abierto (Eoc) vs tiempo de decapado para capilares de acero 316L usados,en una disolución de $\mathrm{NaNO} 3$ al $10 \% \mathrm{~m} / \mathrm{N}$, yHNO3/ HF $24 / 5 \% \mathrm{~m} / \mathrm{v}$.
Para el cálculo de la velocidad de corrosión se hicieron evaluaciones de las pendientes de Tafel para el sistema decapado a 5 y 30 minutos, encontrando como una situación media los siguientes valores de $\beta_{\text {catódico }}=210 \mathrm{mV} / \mathrm{dec}$ y $\beta_{\text {anódico }}=240 \mathrm{mV} / \mathrm{dec}$, lo cual plantea un valor de $B=0,0487 \mathrm{~V}$, según SternGeary (ASME Code, Sectión IX, 2009; ASTM G I02, 20 I0). Estos valores de pendientes de Tafel son elevados, planteando situaciones pasivas en ambos sentidos (anódico y catódico) para el acero 316L en las condiciones de evaluación de decapado.

Mientras que los $\mathrm{E}_{\text {oc }}$ tienden a disminuir, los valores de $V_{\text {corr }}$ tienden a disminuir inicialmente para luego aumentar con el tiempo de decapado, lo cual indicaría que un exceso del tiempo de decapado se torna perjudicial para la superficie, haciéndola más susceptible a la corrosión (ver figuras I y 2). Esto estaría asociado a los efectos contrapuestos planteados para los reactivos decapantes. Los valores plantean que resultaría adecuado un tiempo de decapado de entre 5 y 10 minutos. Para los siguientes ensayos el tratamiento previo fue un decapado por 5 minutos con esta disolución.

\section{Capilares de acero inoxidable AISI-SAE 316L soldados}

Los valores obtenidos en los ensayos electroquímicos con salmuera de un pozo geotérmico se aprecian en el cuadro 4, y aquellos para la disolución de poliacrilato de sodio al $5 \%$ se presentan en el cuadro 5.

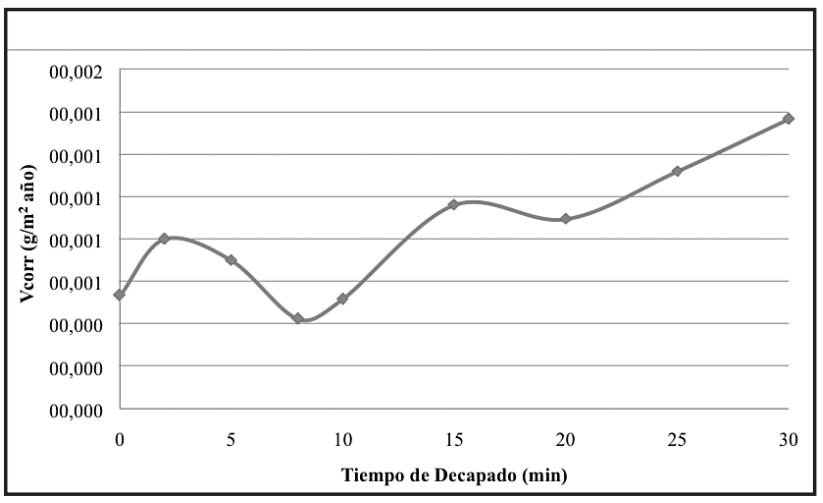

Figura 2. Variación de velocidad de corrosión vs tiempo de decapdo, para capilares de acero 316L usados en una disolución de $\mathrm{NaNO} 3$ al $10 \% \mathrm{~m} / \mathrm{V}, \mathrm{yHNO} 3 / \mathrm{HF} 24 / 5 \% \mathrm{~m} / \mathrm{v}$. 
Cuadro 4.Valores obtenidos en los ensayos con salmuera de pozo geotérmico sobre capilares de acero 316L nuevos, usados, soldados (con y sin decapado) y soldados con TT.

\begin{tabular}{|c|c|c|c|c|c|c|}
\hline Muestra & $\begin{array}{c}E_{o c} \\
(m \vee \text { vs SCE })\end{array}$ & $\begin{array}{c}\mathrm{Rp} \\
\left(\mathrm{W} / \mathrm{cm}^{2}\right)\end{array}$ & $\beta$ cat. $(m V)$ & ßanód. (mV) & $V_{\text {corr }}(\mu \mathrm{m} / a n ̃ o)$ & $\begin{array}{c}V_{\text {corr }}\left(g / m^{2}\right. \\
\text { año) }\end{array}$ \\
\hline Nuevo & $-306,7$ & 365 & 120 & 180 & 0,95 & 7,6 \\
\hline Usado & $-131,0$ & 2448 & 240 & 240 & 0,28 & 2,3 \\
\hline Sin decapar y soldado & $-174,0$ & 55 & 180 & 120 & 9,46 & 75,6 \\
\hline Decapado y soldado & $-205,6$ & 503 & 180 & 240 & 1,63 & 13,1 \\
\hline $\begin{array}{l}\text { Decapado y soldado con } \pi T \\
\text { a } 410^{\circ} \mathrm{C}\end{array}$ & $-154,8$ & 627 & 120 & 210 & 1,36 & 10,84 \\
\hline $\begin{array}{l}\text { Decapado y soldado con } \Pi 1 \\
\text { a } 955^{\circ} \mathrm{C}\end{array}$ & $-282,8$ & 152 & 180 & 150 & 7,26 & 58,04 \\
\hline
\end{tabular}

Cuadro 5. Valores obtenidos en los ensayos electroquímicos con inhibidor de poliacrilato de sodio al $5 \% \mathrm{~m} / \mathrm{m}$ sobre capilares de acero 316L nuevos, usados y soldados (con y sin decapado).

\begin{tabular}{|c|c|c|c|c|c|c|}
\hline Muestra & $\begin{array}{c}E_{o c} \\
(m \vee v s S C E)\end{array}$ & $\operatorname{Rp}\left(W / \mathrm{cm}^{2}\right)$ & $\beta$ cat. (mV) & ßanód. (mV) & $V_{\text {corr }}(\mu \mathrm{m} / a n ̃ o)$ & $\begin{array}{l}V_{\text {corr }}\left(g / \mathrm{m}^{2}\right. \\
\text { año) }\end{array}$ \\
\hline Nuevo & -327 & 2258 & 180 & 150 & 0,20 & 1,6 \\
\hline Usado & 192,5 & 581 & 180 & 240 & 0,93 & 7,5 \\
\hline Decapado y soldado & $-142,2$ & 624 & 270 & 240 & 1,74 & 13,9 \\
\hline Sin decapar y soldado & $-98,06$ & 55 & 180 & 240 & 7,93 & 63,4 \\
\hline $\begin{array}{l}\text { Decapado y soldado con } \\
\pi \text { a } 410^{\circ} \mathrm{C}\end{array}$ & 158,9 & 1717 & 150 & 240 & 0,83 & 6,66 \\
\hline $\begin{array}{l}\text { Decapado y soldado con } \\
\pi \text { a } 955^{\circ} \mathrm{C}\end{array}$ & $-33,22$ & 117 & 150 & 270 & 11,45 & 91,63 \\
\hline
\end{tabular}

En el cuadro 4 los valores de $\mathrm{E}_{\text {oc }}$ muestran una región de trabajo entre la situación de un capilar nuevo y un capilar usado, donde se desarrollarán los potenciales de los capilares soldados, siendo el potencial del capilar soldado y decapado más negativo que aquel sin decapar.

Los capilares soldados con tratamiento tienen una $V_{\text {corr }}$ similar a un capilar nuevo, mientras que los capilares soldados sin tratamiento tienen una $\mathrm{V}_{\text {corr }}$ mucho mayor. Los capilares usados presentan una $V_{\text {corr }}$ menor que un capilar nuevo, posiblemente asociada a la pasivación de la superficie con el uso.

En las curvas de Tafel anódicas de la figura 3 se puede apreciar que las pendientes anódicas para la curva de capilar soldado y sin decapado son mucho menores que para las otras situaciones. Mientras que el valor de la pendiente catódica es menor para el capilar nuevo. El tratamiento térmico a $410^{\circ} \mathrm{C}$ aumenta el $E_{o c^{\prime}}$ sin modificar sustancialmente la $R_{p} y$ variando las pendientes de Tafel, lo que redunda en una Vcorr similar al sistema del capilar decapado y soldado sin tratamiento térmico. En cuanto al tratamiento a $955^{\circ} \mathrm{C}$, aunque mejora el $\mathrm{E}_{o c}$ disminuyendo su valor, disminuye también la $R_{p}$ y las pendientes de Tafel, lo que hace que la $\mathrm{V}_{\text {corr }}$ sea elevada.

En la solución de salmuera se evaluó el efecto de picadura mediante la aplicación de curvas de Tafel anódicas cíclicas, donde en algunos casos puede apreciarse con claridad el potencial de picado $\left(E_{\text {pic }}\right)$, los potenciales de protección $\left(E_{\text {pro }}\right)$, así como los rangos de pasivación $\left(\Delta \mathrm{E}_{\mathrm{pas}}\right)$ y la intensidad $\left(\mathrm{I}_{\mathrm{pas}}\right)$. 


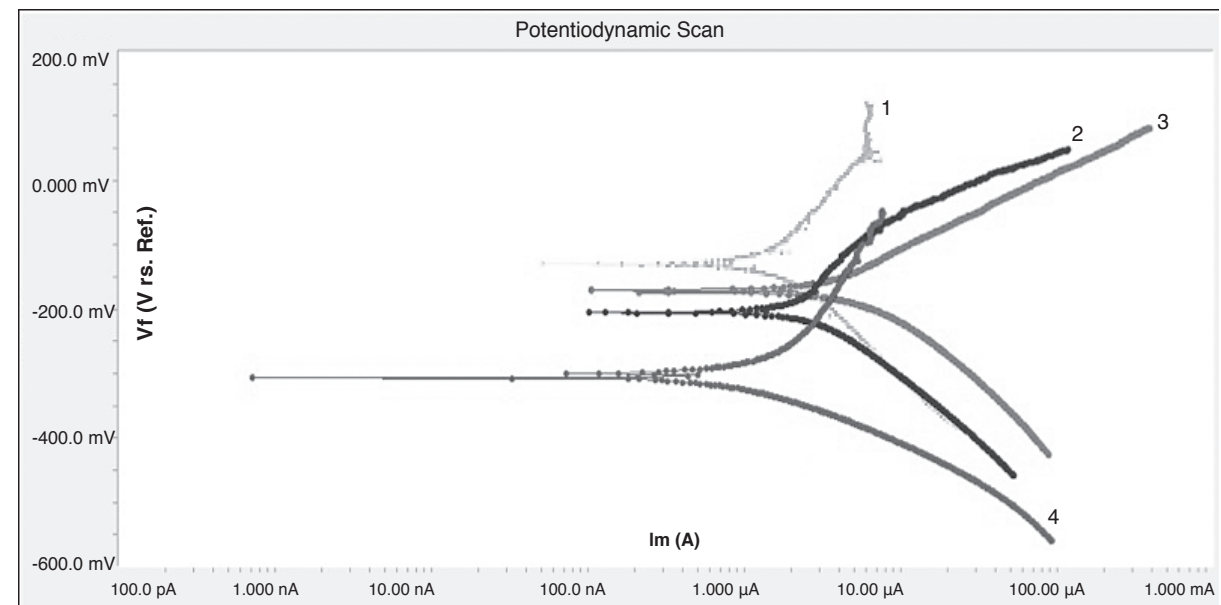

Figura 3. Curvas de Tafel para las distintas muestras evaluadas en disolución de pozo geotérmico, para acero 316L, capilar nuevo (4), capilar usado ( I), capilar decapado y soldado(2), capilar sin decapado y soldado (3).

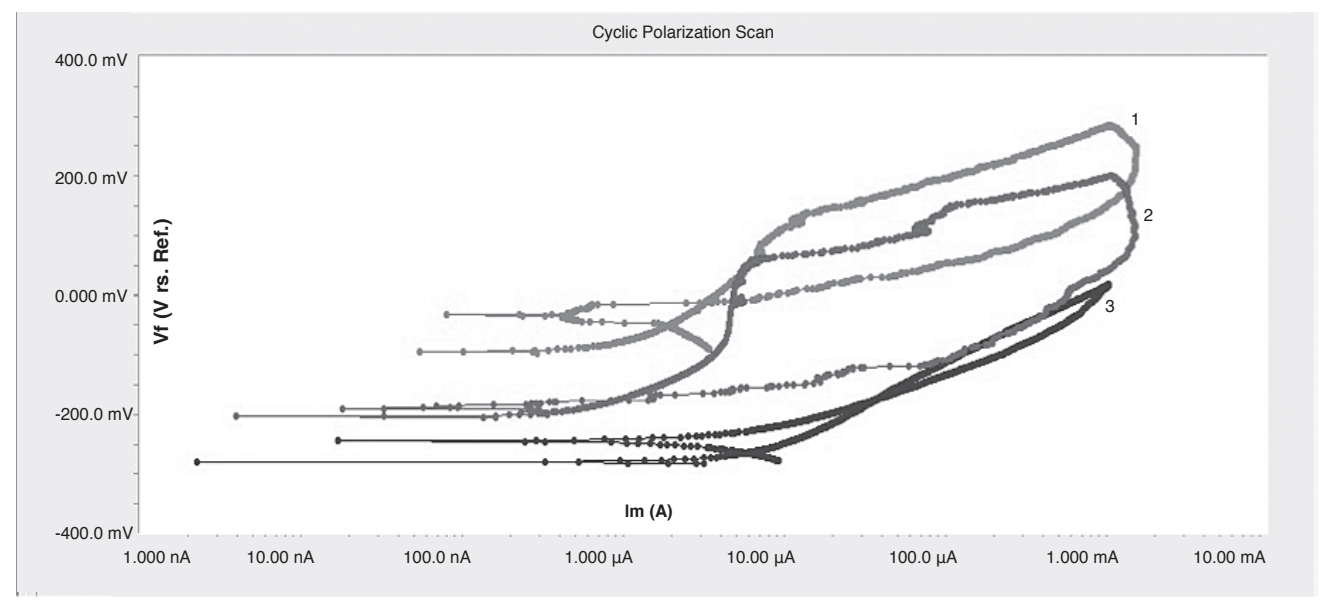

Figura 4. Curvas de Tafel anódicas para las distintas muestras evaluadas en disolución de pozo geotérmico, para acero 316L, capilar soldado con $\pi$ a $410^{\circ} \mathrm{C}$ ( I), capilar soldado con $\pi \mathrm{T}$ a $955^{\circ} \mathrm{C}$ (3), capilar soldado $\sin \Pi \mathrm{T}$ (2).

Es posible observar que la soldadura sin $T$ presenta un rango amplio de $\Delta \mathrm{E}_{\text {pas }}(\mathrm{I} 20 \mathrm{mV})$ con una $\Delta \mathrm{l}_{\text {pas }}$ del orden de I $\mu \mathrm{A}$, lo cual presenta una alta estabilidad frente a la oxidación por picadura con un $\mathrm{E}_{\text {pic }}$ de $+250 \mathrm{mV}$ vs $\mathrm{E}_{\text {oc' }}$ aunque con un $\mathrm{E}_{\text {pro }}$ muy cercano a $\mathrm{E}_{\text {oc }}\left(+25 \mathrm{mV}\right.$ vs $\left.\mathrm{E}_{\mathrm{oc}}\right)$.

El sistema con $\pi \mathrm{T}$ a $410^{\circ} \mathrm{C}$ tiene un comportamiento similar, aunque con un rango de $\Delta \mathrm{E}_{\text {pas }}\left(40 \mathrm{~m}_{\mathrm{v}}\right)$ con un $\mathrm{E}_{\text {pic }}$ menor que sin TT y un $\mathrm{E}_{\text {pro }}$ más alto $(+$ $80 m_{v}$ vs $E_{o c}^{\text {pic }}$ ).

Para el sistema con $\pi \mathrm{T}$ a $955^{\circ} \mathrm{C}$ no se plantea una región definida de pasivación, por lo que no se puede estimar $\mathrm{E}_{\text {pic }^{\prime}}$ y el valor de $\mathrm{E}_{\text {pro }}$ se puede esti- mar en unos $100 m_{v}$ respecto a $E_{o c}$. Mientras que en las curvas catódicas no se aprecia una diferencia sustancial entre los tratamientos.

Los valores de $E_{o c}$ para los sistemas soldados (con y sin decapado) plantean valores intermedios entre los potenciales de un capilar nuevo y uno usado. El sistema decapado y soldado muestra mejor $\mathrm{E}_{\text {oc }}$ respecto al sistema soldado sin tratamiento de limpieza.

Los valores de $V_{\text {corr }}$ plantean que el capilar nuevo tiene una muy baja velocidad de corrosión frente a la disolución de poliacrilato, la cual aumenta progresivamente para el capilar usado y para los capilares soldados, con y sin tratamiento. Este efecto se debe 


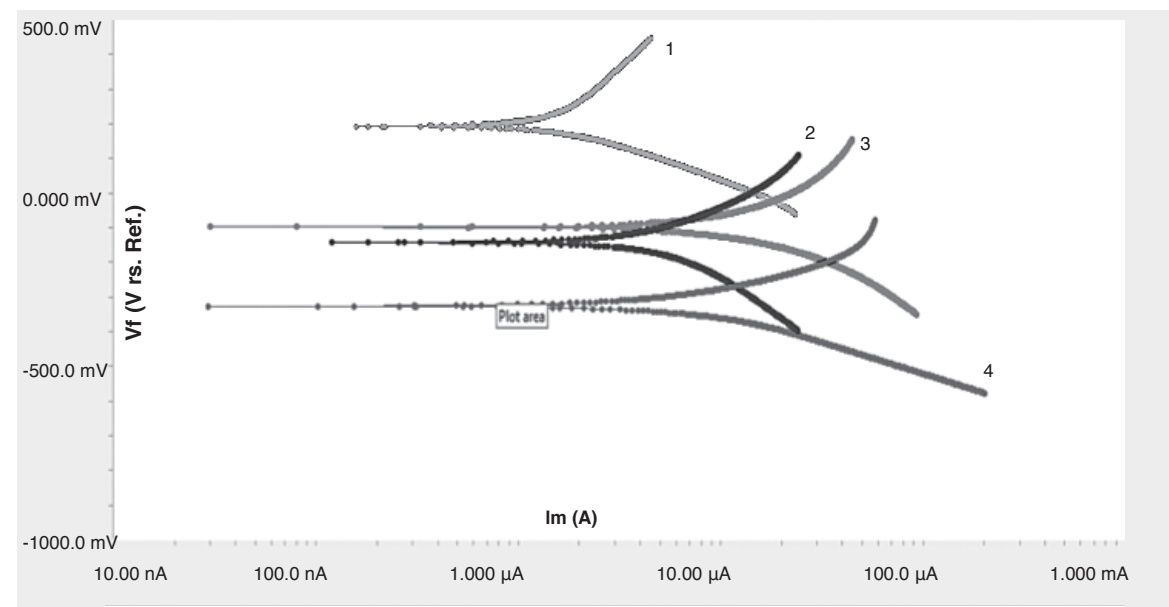

Figura 5. Curvas de Tafel para las distintas muestras evaluadas en disolución de inhibidor de poliacrilato de sodio al 5 \%, para acero 316L, capilar nuevo (4), capilar usado (I), capilar decapado y soldado(2), capilar sin tratar y soldado (3).

a varios factores combinados, partiendo de una disminución progresiva de $R_{p}$ y algunas variaciones en las pendientes de Tafel encontradas (ver figura 5).

El tratamiento térmico a $410^{\circ} \mathrm{C}$ aumenta mucho el $\mathrm{E}_{o c}$ y el Rp, sin variaciones importantes en las pendientes de Tafel, lo que redunda en una $\mathrm{V}_{\text {corr }}$ menor al del capilar decapado y soldado sin tratamiento térmico. Mientras que el tratamiento a $955^{\circ} \mathrm{C}$ aumenta el $\mathrm{E}_{\text {oc }}$ pero disminuye mucho el Rp, lo que hace que la $\mathrm{V}_{\text {corr }}$ sea elevada.

No se observaron sistemas de corrosión por picadura en las Tafel anódicas cíclicas.

\section{Análisis metalográfico}

Mediante análisis metalográfico se observó el acero inoxidable $316 \mathrm{~L}$ previamente al ataque (ver figura 6a), mostrando muy pocas inclusiones no metálicas. Posteriormente al ataque, realizado según la norma ASTM A 407-07 (figura 6b), se corroboró que la soldadura no produjo ningún precipitado de carburos de cromo en los límites de grano y que la estructura es de matriz austenítica para las soldaduras con o sin tratamiento térmico.

Durante las pruebas se sobrepasaron las temperaturas a las cuales ocurre la sensibilización de los aceros inoxidables; sin embargo, no se consideró necesario realizar ensayos de sensibilización, dado que en las metalografías no se determinó la presencia de precipitación de carburos de cromo en los límites de grano, por lo cual se evidencia que los capilares no sufrieron sensibilización.
Además, el proceso de soldadura utilizado para la unión de tubo capilar imposibilita el proceso de sensibilización, ya que el calor generado en el proceso es mínimo. Asimismo, la escasa cantidad de carbono presente en la aleación dificulta la formación de carburos de cromo.

\section{Conclusiones}

El proceso de decapado plantea un rango de tiempos óptimos asociados a la limpieza de óxidos superficiales, sin generar un deterioro importante de la superficie y disminuyendo su $\mathrm{V}_{\text {corr }}$ Los tiempos sugeridos son de 5 a 10 minutos. Aquellos capilares que se decaparon durante un tiempo mayor presentan un $\mathrm{E}_{o c}$ más negativo, pero se nota un incremento en las $\mathrm{V}_{\text {corr' }}$ debido al ataque del HF sobre la muestra.

Los ensayos electroquímicos en las soluciones de trabajo indican que comparativamente ambos medios plantean valores de $V_{\text {corr }}$ similares para el capilar decapado y soldado; estos siempre fueron menores cuando se comparan con los capilares donde no se aplicó tratamiento de limpieza. Es clara la desventaja cuando se comparan los datos de las muestras decapadas y soldadas frente a los capilares nuevos en cuanto a $E_{o c} y V_{\text {corr }}$. Mientras que los capilares usados no brindan valores más beneficiosos de $\mathrm{E}_{o c}$ pero sí de $\mathrm{V}_{\text {corr }}$ debido probablemente a la capa pasiva generada en su superficie cuando se utilizó en los pozos geotérmicos.

Los tratamientos térmicos no mejoran sustancialmente los valores de $V_{\text {corr }}$ para justificar su aplicación, 
a)
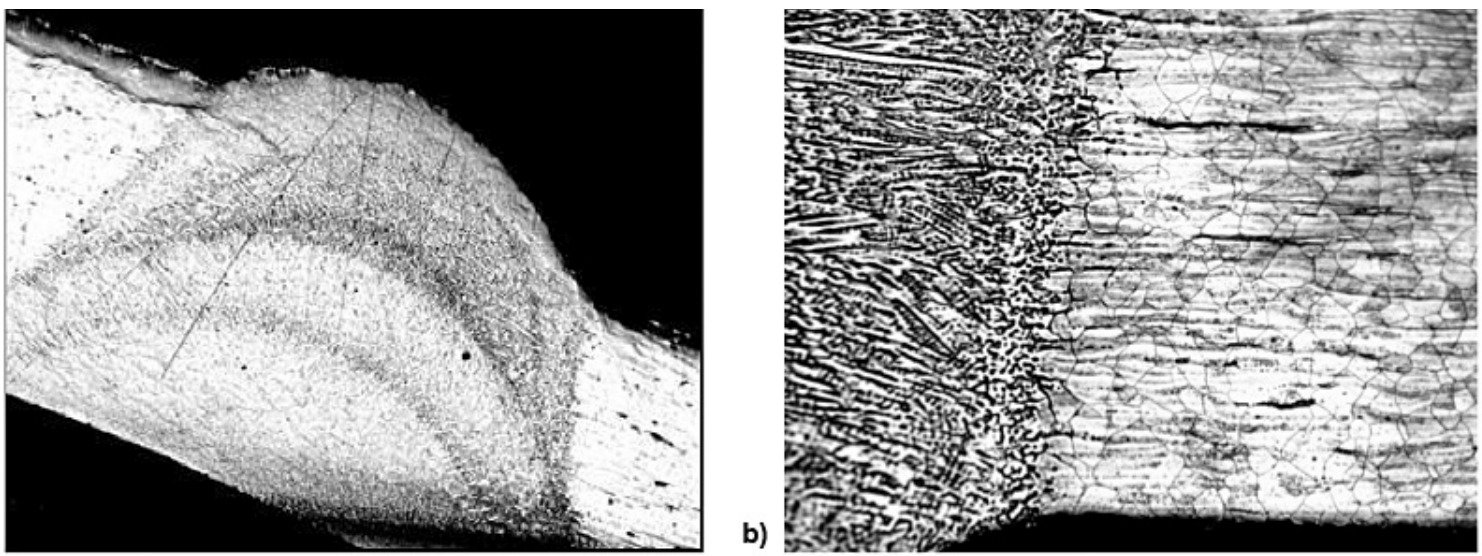

Figura 6. Soldadura de tubo capilar AISI $316 \mathrm{~L}, \mathrm{a})$ sin atacar, $\times 50$, b) soldadura tratada térmicamente a $955^{\circ} \mathrm{C}$, atacada con disolución de ácido oxálico, ×200

pero se debe analizar su efecto sobre la reducción de tensiones debido a la soldadura. Además, las metalografías plantean que el proceso de soldadura y los tratamientos térmicos no presentan problemas de precipitación del cromo o carburos en el cordón de soldadura y las zonas adyacentes.

\section{Agradecimientos}

Se agradece de forma especial el apoyo brindado por el Centro de Servicios y Recursos Geotérmicos del Instituto Costarricense de Electricidad (ICE) para el desarrollo de este proyecto.

\section{Bibliografía}

ASM International Metal Handbook (1990). Heat Treating, US. (4).

ASTM A 380-06 (2006) Standard Practice for Cleaning, Descaling, and Passivation of Stainless Steel Parts, Equipment, and System.

ASTM A 407-07 (2007) Standard Practice for Microetching Metals and Alloys.

ASTM D I193-06. (201 I). Standard Specification for Reagent Water.

ASTM G 102-89. (2010). Standard Practice for Calculation of Corrosion Rates and Related Information from Electrochemical Measurements.

Bard, A. \& Faulkner, L. (200 I). Electrochemical Methods. New York: J.Wiley \& Sons.

Código ASME, Sección IX (2009). Soldadura: Desarrollo y calificación de Procedimientos y Soldadores. Sociedad Americana de Ingenieros Mecánicos
Davies, J. et. al. (1998). Metals Handbook. ASM International, Ohio. US.

Ellis, P. (1985). Companion study guide to short course on geothermal corrosion and mitigation in low temperature geothermal heating systems. Texas.

Fontana, M. (1986). Corrosion Engineering. 3 ed. US: Mac Graw Hill.

González, J. (1989). Control de la Corrosión: Estudio y Medida por Técnicas Electroquímicas. Madrid: Centro Nacional de Investigaciones Metalúrgicas.

Laufgang, S. (2000). Aceros Inoxidables. Universidad de Buenos Aires. Disponible en: http://materias.fi.uba.ar/6750/ ACEROS\%20INOXIDABLES.pdf

Mora Rodríguez, J. (2006). Programa de control del tubo capilar usado en los pozos del Campo Geotérmico Miravalles. Tesis de Maestría en Administración de la Ingeniería Electromecánica, Instituto Tecnológico de Costa Rica.

Mundhenk, N., Huttenloch, P., Zorn, R., \& Kohl, T. (20/3). Electrochemical study on corrosion of different metals exposed to deaerated geothermal brines containing $\mathrm{CO} 2$. Institute of Applied Geosciences, Alemania.

Refaey, S. A. M., Taha, F., \& El-Malak, A. A. (2006). Corrosion and Inhibition of $316 \mathrm{~L}$ stainless steel in neutral medium by $2-\mathrm{M}$ ercaptobenzimidazole. International Journal of Electrochem. Science, I, 80-9|

Schweitzer, P.A. (1986). Corrosion Resistance Tables. US: Marcel Dekker.

Whittaker, P. (2009). Corrosion in the Kalina Cycle. Tesis de Maestría,The School for Renewable Energy Science, Islandia.

XU, C., ZHANG, Y., CHENG, G., \& ZHU,W. (2006). Corrosion and electrochemical behavior of $316 \mathrm{~L}$ stainless steel in sulfate-reducing and iron-oxidizing bacteria solutions. Chinese Journal of Chemical Engineering, 14(6), 829-834. 